\title{
Other Iatrogenic Immunodeficiency-associated Lymphoproliferative Disorder, Hodgkin Type, following Epstein-Barr Viral Hepatitis in a Patient with Rheumatoid Arthritis
}

Masuho Saburi ${ }^{1,2}$, Masao Ogata ${ }^{1}$, Natsumi Yoshida ${ }^{1}$, Yuko Nashimoto ${ }^{1}$, Yui Moroga ${ }^{1}$, Kuniko Takano ${ }^{1}$, Kazuhiro Kohno ${ }^{1,2}$, Tsutomu Daa $^{3}$ and Kuniaki Shirao ${ }^{1}$

\begin{abstract}
:
A 59-year-old man with an 18-year history of rheumatoid arthritis who had been treated with steroids, methotrexate, and infliximab presented with a high-grade fever, cervical lymphadenopathy, and hepatosplenomegaly. Epstein-Barr virus (EBV) hepatitis was diagnosed based on the liver histology and EBV antibody titer. The symptoms improved temporarily, but five months later, the fever, skin rash, jaundice, and thrombocytopenia relapsed. Bone marrow and liver biopsies demonstrated infiltration with Reed-Sternberg cells. Based on these findings, the patient was diagnosed with other iatrogenic immunodeficiency-associated lymphoproliferative disorder (OIIA-LPD), Hodgkin lymphoma type. This case followed a rare clinical course, in that acute hepatitis preceded the diagnosis of OIIA-LPD.
\end{abstract}

Key words: other iatrogenic immunodeficiency-associated lymphoproliferative disorder, Hodgkin lymphoma, Epstein-Barr virus

(Intern Med 57: 1145-1149, 2018)

(DOI: 10.2169/internalmedicine.9599-17)

\section{Introduction}

Other iatrogenic immunodeficiency-associated lymphoproliferative disorder (OIIA-LPD) typically develops in patients with autoimmune diseases who are receiving methotrexate (MTX) and other biological drugs such as infliximab (IFX) (1). Epstein-Barr virus (EBV) may also have a causative role, especially in Hodgkin lymphoma-type OIIALPD $(2,3)$. We herein describe a case of OIIA-LPD, Hodgkin lymphoma type, following EBV hepatitis. This case followed a rare clinical course in that acute hepatitis preceded a diagnosis of OIIA-LPD.

\section{Case Report}

A 59-year-old man developed rheumatoid arthritis (RA) in 1996 and had been treated with prednisolone (PSL) and
MTX. IFX was started from 2006 in combination with PSL and MTX. In 2014, he presented with high-grade fever. Ten days later, he was examined in a clinic and was found to have slight anemia and increased hepatobiliary enzyme levels; viral capsid IgM antibody (VCA-IgM) [enzyme immunoassay (EIA)] was detected (2.4). He was referred to our hospital because of prolonged symptoms, which had persisted for 3 weeks after the onset of symptoms. Computed tomography (CT) showed mild para-aortic lymphadenopathy and hepatosplenomegaly. MTX and IFX were discontinued. A histological examination of a liver specimen revealed inflammatory cell infiltration. The laboratory findings showed no evidence of hepatitis A, B, or C, cytomegalovirus, druginduced liver injury, or autoimmune hepatitis. The patient's EBV antibody titers [fluorescence antibody method (FA)] were as follows: viral-capsid antigen $(\mathrm{VCA}-\operatorname{IgM})<1: 10$; viral capsid IgG antibody (VCA-IgG) 1:320; and Epstein-Barr nuclear antibody (EBNA) <1:10. EBV hepatitis was diag-

${ }^{1}$ Department of Medical Oncology and Hematology, Oita University Faculty of Medicine, Japan, ${ }^{2}$ Department of Hematology, Oita Kouseiren Tsurumi Hospital, Japan and ${ }^{3}$ Department of Diagnostic Pathology, Faculty of Medicine, Oita University, Japan Received: June 1, 2017; Accepted: August 19, 2017; Advance Publication by J-STAGE: December 27, 2017 Correspondence to Dr. Masuho Saburi, masuho-saburi@oita-u.ac.jp 


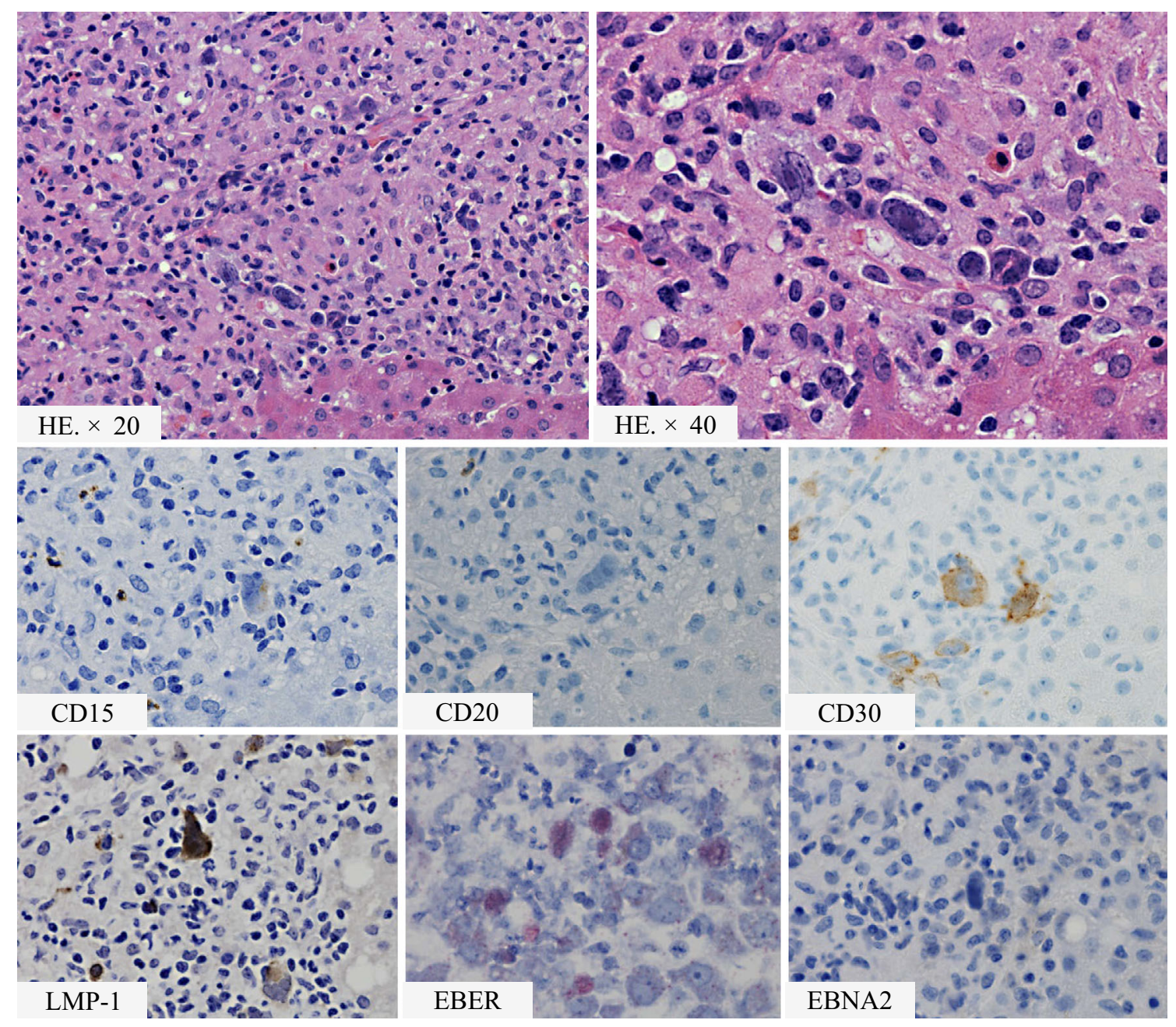

Figure 1. Liver specimens were subjected to Hematoxylin and Eosin staining (original magnification $\times 20, \times 40$ ), immunohistochemical studies, and in situ hybridization for EBV-encoded RNA (EBER-ISH). Large, atypical nucleated cells were seen in the nodules. The large cells were similar in appearance to Reed-Sternberg (RS) cells and were positive for CD30 and latent membrane protein 1 (LMP-1) and negative for CD20, CD15, and EBV nuclear antigen 2 (EBNA2) on immunostaining. EBER-ISH detected RS cells.

nosed based on the liver histopathology and VCA-IgM (EIA) positivity, in addition to the other EBV antibody titers (FA). The symptoms temporarily improved without any treatment within 1 month after the onset, and his RA remained stable with PSL alone. At five months after the diagnosis of EBV hepatitis, the patient developed high-grade fever, skin rash, and jaundice. The laboratory findings included increased biliary enzymes, anemia, and thrombocytopenia. CT showed the worsening of the para-aortic lymphadenopathy and hepatosplenomegaly. The soluble interleukin-2 receptor level was $20,665 \mathrm{U} / \mathrm{mL}$, and the EBV antibody titer demonstrated a previous infection pattern (VCA-IgM <1:10, VCA-IgG 1:640, EBNA 1:10). The EBVDNA level was $<20$ copies $/ 10^{6}$ cells in the peripheral blood. A pathological examination of a liver specimen revealed epithelioid granuloma composed mainly of CD68a-positive histiocytes and CD3-positive $\mathrm{T}$ cells (CD4> CD8) within the expanded portal region of the liver parenchyma, and a few large, atypical nucleated cells were detected in the nodules
(Fig. 1). The large cells were similar in appearance to ReedSternberg (RS) cells and were positive for CD30 and latent membrane protein 1 (LMP-1), and negative for CD79a, CD 20, CD15, epithelial membrane antigen (EMA), and EBV nuclear antigen 2 on immunostaining. In situ hybridization for EBV-encoded RNA (EBER) detected RS cells. The pathological examination of a bone marrow clot showed the infiltration of lymphocytes around large cells with abnormal large multilobular nuclei (Fig. 2). The immunostaining and EBER profiles of these large cells were similar to the RSlike cells of the liver. Immunostaining of the lymphocytes around these cells revealed that they were positive for $\mathrm{CD} 3$, CD4 and CD8 (CD4> CD8), and negative for CD20 and CD 79a. A pathological examination of a skin specimen showed no abnormal cellular infiltration. Due to the history of MTX and IFX use, OIIA-LPD (classical Hodgkin lymphoma-type, Stage IVB) was diagnosed in conformity with the fourth edition of the WHO classification. He had been treated with methylprednisolone (mPSL; $250 \mathrm{mg}$ ) for 3 days before the 


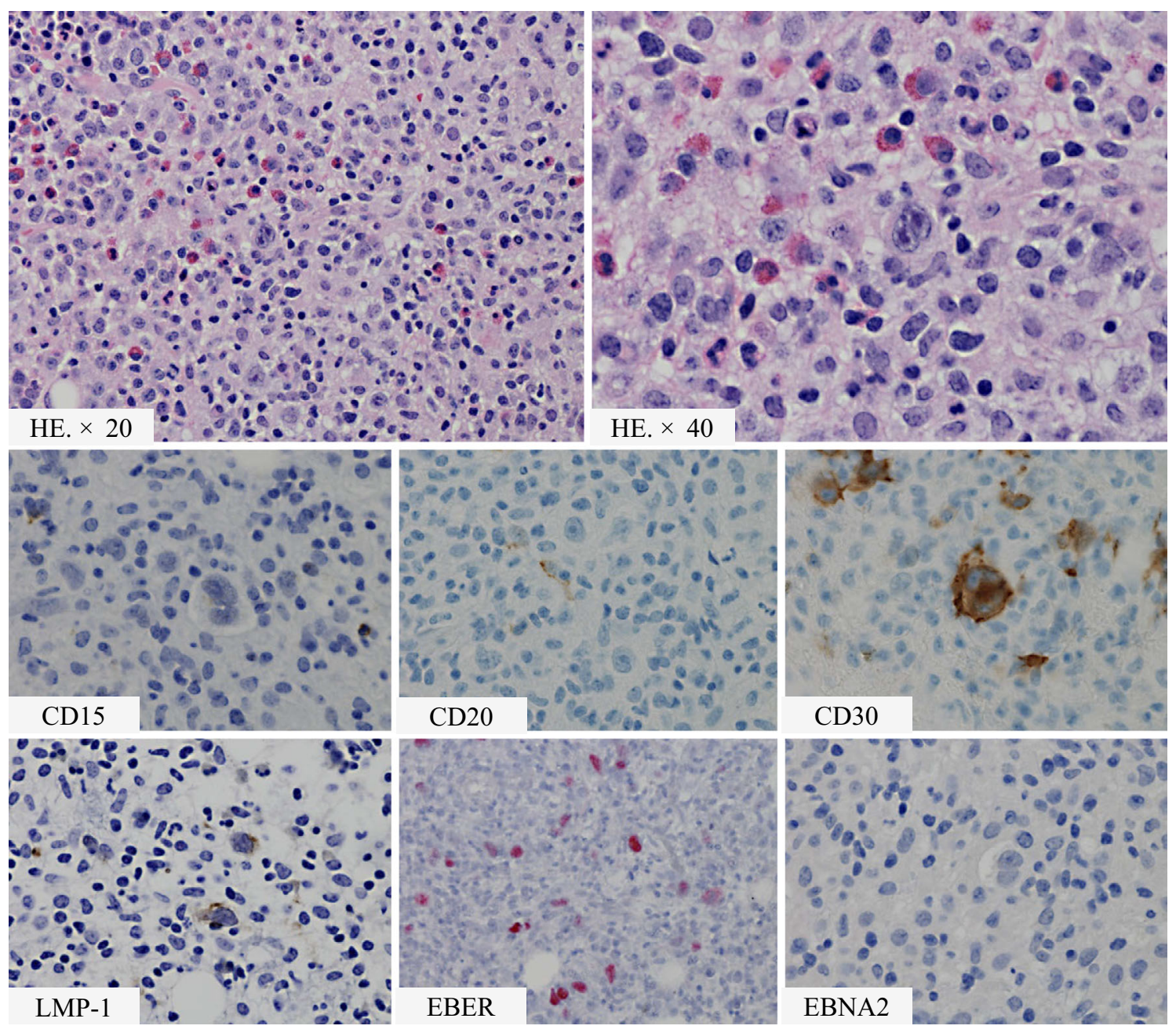

Figure 2. Bone marrow clot specimens were subjected to Hematoxylin and Eosin staining (original magnification $\times \mathbf{2 0}, \times \mathbf{4 0}$ ), immunohistochemical studies, and in situ hybridization for EBV-encoded RNA (EBER-ISH). The infiltration of lymphocytes was observed around large cells with abnormal, large, multilobular nuclei, which were similar in appearance to Reed-Sternberg (RS) cells. These cells were positive for CD30 and latent membrane protein 1 (LMP-1), and were negative for CD20, CD15, and EBV nuclear antigen 2 (EBNA2) on immunostaining. EBER-ISH detected RS cells.

diagnosis of OIIA-LPD (Fig. 3). His fever waned, and the skin rash disappeared immediately after the administration of mPSL. Thrombocytopenia and the increased biliary enzyme levels improved but persisted. After the diagnosis of OIIA-LPD, he was treated with ABVD (adriamycin, bleomycin, vinblastine, and dacarbazine), and the mPSL dose was tapered. After 1 course of ABVD, his platelet count and hepatobiliary enzymes normalized. After 2 courses of ABVD, he reached complete remission (CR); a total of 6 courses were administered. At two years after chemotherapy, he remains in CR. The patient is currently treated with lowdose prednisone and tacrolimus; there have been no exacerbations of RA with this regimen.

\section{Discussion}

The present patient developed EBV hepatitis while being treated for RA with MTX and IFX. Although his symptoms improved temporarily, OIIA-LPD developed in the form of
Hodgkin lymphoma with increased biliary enzymes and thrombocytopenia at 5 months after the diagnosis of EBV hepatitis. OIIA-LPD is a heterogeneous group of diseases classified by a history of using immunosuppressive medication such as MTX in the setting of autoimmune disease. Diffuse large B-cell lymphoma and Hodgkin lymphoma are two major histological subtypes of OIIA-LPD. Fifty-four cases of OIIA-LPD have been reported in patients with the form of classical Hodgkin lymphoma (2). The administration of MTX for the treatment of RA was the most frequent patient characteristic. Lymphadenopathy was the common disease site, but two cases of bone marrow and two cases of liver involvement have also been reported (2). The frequency of EBV infection (EBER-positive and/or LMP-1-positive) was $80 \%$, which is higher than that in patients with sporadic classical Hodgkin lymphoma (approximately 40\%). Many cases require cytotoxic chemotherapy for treatment, as well as the discontinuation of immunomodulatory drugs $(2,3)$. The clinical features of the present case were consistent with 


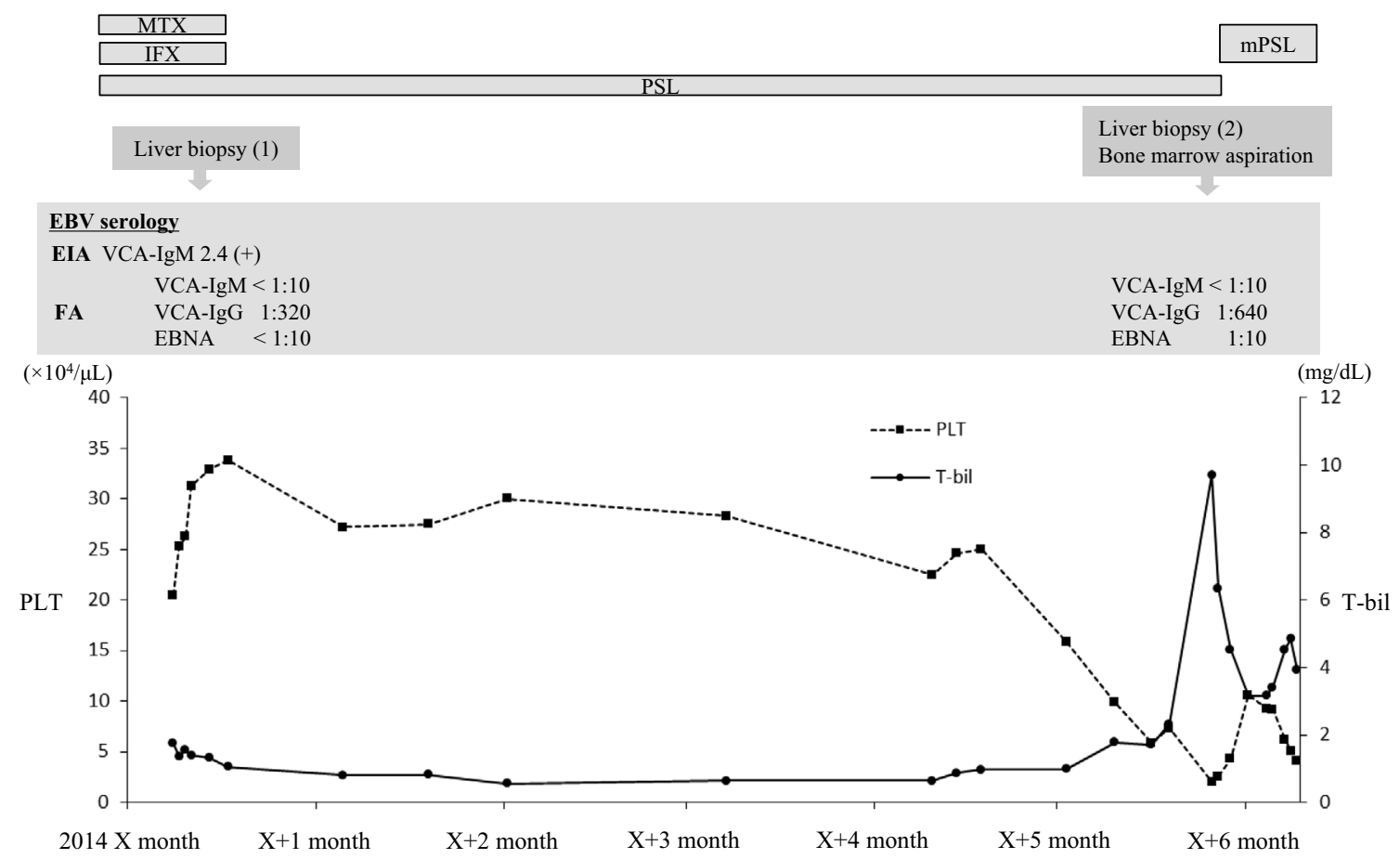

Figure 3. The clinical course from EBV hepatitis to the diagnosis of malignant lymphoma. PLT: platelet count, T-bil: total bilirubin, VCA-IgM: viral capsid IgM antibody, VCA-IgG: viral capsid IgG antibody, EBNA: Epstein-Barr nuclear antibody, EIA: enzyme immunoassay, FA: fluorescence antibody method, MTX: methotrexate, IFX: infliximab, PSL: prednisolone, mPSL: methylprednisolone

those of previous reports, but the clinical course was rare, in that acute hepatitis preceded the diagnosis of OIIA-LPD. As for the patient's acute hepatitis, two possibilities were considered based on the clinical course and laboratory findings.

The first possibility is that the liver involvement of OIIALPD went undiagnosed in the histological examination of the first liver biopsy specimen. The liver biopsy was performed by needle biopsy; thus, the specimen might not have contained abnormal lesions. In addition to the possible sampling error, the clinical course of the temporary improvement after the discontinuation of MTX and IFX fits the response to therapy for OIIA-LPD Hodgkin lymphoma. Two cases of Hodgkin lymphoma have been reported in RA patients with antecedent symptoms of suspected MTXLPD (4). In this report, the antecedent symptoms improved temporarily with the discontinuation of MTX or steroid pulse therapy but were exacerbated after 1.5 years with aggressive manifestations. In one of the cases, the patient was not diagnosed by a liver biopsy and was only diagnosed at autopsy. It is unclear whether the antecedent symptoms were due to OIIA-LPD because no malignant cells were detected in the histological examination. However, similarly to our case, the antecedent symptoms were not diagnosed at the time of the first biopsy. We hypothesize that OIIA-LPD might sometimes become exacerbated with aggressive manifestations after temporary improvement following the discontinuation of immunosuppressive drugs.

The second possibility is that EBV hepatitis was the pri- mary EBV infection. More than $90 \%$ of the world's adult population is infected with EBV (5). Thus, primary EBV infection would be rare in this 59-year-old patient. VCA-IgM sometimes increases with reactivation and is associated with false-positive results. In addition, there were no stored serum samples from before the manifestation of hepatitis; thus, it was not possible to prove the EBV seronegative status. However, EBV primary infection was considered because of seroconversion of EBNA and the results of the histological examination of the first liver specimen. The occasional viral lytic cycle is also thought to play a pivotal role in the development and maintenance of tumors because of its association with the secretion of cytokines and growth factors $(6,7)$. Especially in renal transplantation, it has been reported that EBV-related posttransplantation lymphoproliferative disorder (PTLD) developed within a short period of time after primary EBV infection. EBV-seronegative patients who received a renal allograft from EBV-seropositive donors were at risk of developing EBV-related PTLD (8-10). Since primary EBV infection is a risk factor for LPD in a compromised host, it may be necessary to confirm a serological analysis to determine a patient's EBV status before the administration of immunosuppressive drugs such as MTX.

In conclusion, OIIA-LPD, Hodgkin lymphoma-type, developed after EBV hepatitis in a patient with RA. If the symptoms of suspected MTX-LPD are seen with no histological evidence of malignancy in RA patients who are on immunosuppressive treatment, the patient should be care- 
fully followed to identify the potential exacerbation of undiagnosed LPD and to check their EBV status, while considering the possibility of primary infection with EBV.

The authors state that they have no Conflict of Interest (COI).

\section{References}

1. Tokuhira M, Watanabe R, Nemoto $T$, et al. Clinicopathological analyses in patients with other iatrogenic immunodeficiencyassociated lymphoproliferative diseases and rheumatoid arthritis. Leuk Lymphoma 53: 616-623, 2012.

2. Loo EY, Medeiros LJ, Aladily TN, et al. Classical Hodgkin lymphoma arising in the setting of iatrogenic immunodeficiency: a clinicopathologic study of 10 cases. Am J Surg Pathol 37: 12901297, 2013.

3. Carbone A, Spina M, Gloghini A, Tirelli U. Classical Hodgkin's lymphoma arising in different host's conditions: pathobiology parameters, therapeutic options, and outcome. Am J Hematol 86: 170-179, 2011.

4. Tokuhira M, Tabayashi T, Tanaka Y, et al. The aggressive clinical courses of Hodgkin lymphoma primarily diagnosed as methotrexate-induced non-specific lymphoproliferative disorder in patients with rheumatoid arthritis. J Clin Exp Hematop 56: 165-
$169,2017$.

5. Andersson J. Epstein-Barr virus and Hodgkin's lymphoma. Herpes 13: 12-16, 2006

6. Murata T, Sato Y, Kimura H. Modes of infection and oncogenesis by the Epstein-Barr virus. Rev Med Virol 24: 242-253, 2014.

7. Murata T, Tsurumi T. Switching of EBV cycles between latent and lytic states. Rev Med Virol 24: 142-153, 2014.

8. Hosseini-Moghaddam SM, Alhomayeed B, Soliman N, Weir MA, House AA. Primary Epstein-Barr virus infection, seroconversion, and post-transplant lymphoproliferative disorder in seronegative renal allograft recipients: a prospective cohort study. Transpl Infect Dis 18: 423-430, 2016.

9. Leaver S, Amlot $\mathrm{P}$, Thuraisingham R, Norton A, Aitken C, Cavenagh JD. Subacute immune response to primary EBV infection leading to post-transplant lymphoproliferative disease in a renal transplant patient. Clin Lab Haematol 26: 351-353, 2004.

10. Schachtner T, Reinke P. Pretransplant prophylactic rituximab to prevent Epstein-Barr virus (EBV) viremia in EBV-seronegative kidney transplant recipients from EBV-seropositive donors: results of a pilot study. Transpl Infect Dis 18: 881-888, 2016.

The Internal Medicine is an Open Access article distributed under the Creative Commons Attribution-NonCommercial-NoDerivatives 4.0 International License. To view the details of this license, please visit (https://creativecommons.org/licenses/ by-nc-nd/4.0/).

(C) 2018 The Japanese Society of Internal Medicine

Intern Med 57: 1145-1149, 2018 